\title{
PRESUNTOS MÉTODOS MAYAS DE CÁLCULOS CRONOLÓGICOS
}

\author{
Por César Lizardi Ramos \\ Seminario de Cultura Maya. \\ U.N.A.M.
}

El propósito que anima al autor de este artículo es dar pruebas, siquiera parciales e indirectas, de que no pocos de los métodos usados hoy día por los cronólogos mayistas pudieron haber sido conocidos y usados por los cronólogos mayas, quienes, muy probablemente, conocieron otros muchos, de que nosotros no tenemos ni la menor idea.

El método seguido consiste en mostrar que las operaciones relativas a la cronología maya resultan de una facilidad extremada si aplica uno determinadas reglas emanadas de la observación adecuada de ciertas cualidades del calendario mismo. Esa facilidad llega inclusive a hacer, de una operación de aquéllas, un juego pueril y sirve de justificación, o a lo menos de puntal, a la idea de que así como nosotros la aprovechamos, pudieron haberla aprovechado también los admirables cronólogos mayas.

Para realizar su designio, el autor presenta una numerología que es una "llave para acertar y hallar" fechas, distanciastiempo y posiciones, ora en los ciclos del Calendario maya, ora en la Cuenta Larga.

Algunas partes de semejante numerología - por ejemplo, la relativa a la Regla del Baktún Doble - muestran un paralelismo notable con la Regla del Uinal Doble, usada y comentada, entre otros, por los doctores Zimmermann (1935), Thompson (1941) y Satterthwaite (1947).

Si bien esas partes no parecen haber sido descritas por otros investigadores, no se pretende aquí reivindicar la paternidad sobre ellas, pues el autor piensa que en el campo de los números no hay descubrimientos exclusivos, ya que esos números se dan de todo a todo a quienquiera que sepa interrogarlos. 
Pregunta renovada y frecuente en los labios y la pluma de los investigadores mayistas es la multiforme que inquiere si los mayas antiguos conocían tal o cual noción, tal o cual procedimiento que nosotros creemos descubrir en sus inscripciones o en sus libros jeroglíficos y que nos parece trascendental, original, ingenioso, y por ello mismo, propio para ser encontrado por humanos sabios como nosotros, no por gente primitiva de cuyo genio e inventiva dudamos, pese al hecho de que esa gente creó, inventó, o mejoró, los sistemas mismos que causan nuestro asombro y que excitan, por emulación, nuestros recursos mentales y nos conducen a hacer hallazgos que luego damos en considerar como demasiado sutiles y encumbrados para la inteligencia de aquellos antiguos.

No deja de haber, por cierto, un rasgo plausible en esta duda nuestra, rasgo que consiste en la timidez con que todo hombre de ciencia debe proceder cuando se trata de sentar hechos, formar juicios y sacar conclusiones.

Pero al mismo tiempo, a lo que pienso, echa uno de ver que está pasando por alto aquel modo realista de ver las cosas que nos obliga a comprender que lo que hace un individuo puede hacerlo otro individuo, circunstancia que puso de manifiesto con franqueza cruda, y acaso agresiva, un matemático inglés que hace más de un cuarto de siglo, si no claudica mi memoria, publicó un manualito de matemáticas, movido por el propósito de explicar lisa y meridianamente, los fenómenos de la matemática elemental, inclusive con ejemplos muy familiares.

El sagaz y francote británico puso la frase siguiente como epígrafe de su simpática obra - simpática a pesar de la opinión adversa, pero justiciera, que formuló acerca de algunos de sus puntos débiles un sabio mexicano, ahora finado:

"What a fool does, any other fool can do."

Impresionado el que esto escribe por el modo de ver del autor británico, y consciente de que los cronólogos mayas pudieron haber hecho, por lo menos, muchas de las observaciones que hemos hecho nosotros en sus sistemas y haber usado métodos equivalentes a varios de los nuestros, y consciente, por añadidura, de que en el campo de la astronomía y la matemática pudieron haberse metido aun en terrenos que nosotros no sospechamos siquiera que hubieran hollado, ha determinado hacer relación de muchos procedimientos, operaciones, observacio- 
nes, que conocen los cronólogos mayistas, y poner en relieve su sencillez, para inferir de allí, o a lo menos para insinuar, que los sabios mayas, que manejaban sistemas creados o inventados por sus maestros y por ellos mismos y sus discípulos, pudieron haber practicado, con el mismo, o mayor conocimiento de causa y con la misma, o mayor sagacidad, muchas de las operaciones que nosotros ejecutamos hoy día cuando estudiamos, o comentamos, la sabiduría de los mayas antiguos. Y ha resuelto el autor de estas líneas, por ende, hacer hincapié en que muchas de esas operaciones pueden ser ejecutadas de memoria, o casi, lo cual hará ver que es grande la posibilidad de que los mayas las hayan practicado también, puesto que se trata de cosas sencillas y fáciles. Pero aun hay más: existen en esas operaciones nuestros caminos obligados, cosas del sentido común más universal, circunstancias que pueden militar en favor de la idea de que los sacerdotes mayas, muchos de ellos, probablemente, doctos en su ciencia cronológica y hábiles en su aplicación, las ejecutaban habitualmente.

Típicas de la escéptica, o agnóstica actitud de los mayistas, me parecen las palabras del doctor John E. Teeple (1935:60, Separata):

"Pero ¿lo sabían los mayas? He aquí el punto que no es claro para nosotros". Palabras que vienen a continuación de éstas, relativas a Copán: "La Estela 19 da una fecha 9.10.19. 15.0, 4 Ahau 8 Chen, que, sabemos, es el $3,765^{\circ}$ aniversario de 4 Ahau 8 Cumkú".

Duda digna de atención, pero acaso catalogable en el grupo de las que vengo comentando, es la que expresa Linton Satterthwaite Jr. (1947:48) al afirmar que las Tablas de Ola Ápenes (1939) "hermosamente compactas pero sin embargo complicadas" ("elaborate") son ciertamente de los procedimientos que no pretenden estar cortados por el patrón de "probables métodos mayas". No quiero dejar pasar la oportunidad de manifestar que a mi juicio, algunos de los métodos aplicados por Ápenes en esas tablas, pueden ser trasuntos, o equivalentes, de los seguidos por los propios mayas en el terreno de la cronología. Acaso en otra parte me sea dada la oportunidad de explicar mi modo de pensar a este respecto, apoyado en argumentos e indicios.

Al recordar muchos de los procedimientos y hallazgos de los mayistas insistiré en la viabilidad y aun facilidad de aqué- 
llos que podemos ejecutar de memoria, para hacer resaltar, por analogía, la posibilidad de que los mayas mismos hicieran lo propio, o cosa semejante, y acaso con éxito mejor que el nuestro, no obstante la amplitud, eficacia y novedad de la técnica al alcance de nuestra mano.

En conclusión, repito que lo que hago aquí es insinuar que lo que nosotros ejecutamos con facilidad, pudieron haberlo hecho también los cronólogos mayas.

\section{ARSENAL DE LOS MAYISTAS}

Cualquier cronólogo mayista es capaz de hacer mentalmente, o con ayuda de apuntamientos muy escasos, un cúmulo de cálculos cronológicos, a veces harto complicados y curiosos, pero por lo común, más bien aparatosos, aunque eficaces.

$\mathrm{Y}$ es capaz de todo esto, porque sabe de memoria:

a) La lista de los $20 \mathrm{~K}$ 'ines, y sus números de orden dentro de la veintena, de modo que puede indicar sin tardanza ni vacilación, que Ben es el día $13^{\circ}$, que Eznab es el $18^{\circ}$, que Cimi es el $6^{\circ}$, etc.

b) La lista de los 18 Uinales, y su orden respectivo, de manera que puede informarnos que Zac es el $11^{\circ}$, que $\mathrm{K}^{\prime}$ ayab es el $17^{\circ}$, que Xul es el $6^{\circ}$, etc. dario.

c) La lista de los 52 años del Ciclo, o Rueda de Calen-

d) Las series de los números que afectan las repeticiones sucesivas de un mismo $\mathrm{K}$ 'in a intervalos iguales a los períodos de la Cuenta Larga.

e) Los 10 múltiplos primeros, cuando menos, del 13 .

f) Un cúmulo de peculiaridades, al parecer curiosas, pero de hecho muy importantes, del sistema cronológico de los mayas, por ejemplo: los intervalos con que se repiten las Fechas de Rueda de Calendario en una posición dada de la serie de los Tunes, o de los Katunes, o de los Baktunes, etc., o bien, fenómenos como la repetición, cada cuatro años, de un mismo $\mathrm{K}$ 'in, pero con un numeral diferente, que ocupa determinada posición en el Haab; o todavía, el modo de calcular posiciones de las Fórmulas Diurnas, o de las Fechas de Rueda de Calendario en años dados, o de calcular el año a que pertenece una Fecha de Rueda de Calendario. 
TZOLKIN

\begin{tabular}{|c|c|c|c|c|c|c|c|c|c|c|c|c|}
\hline 1-Imix & 8-1mix & $2-I_{\operatorname{mix}}$ & 9. Imix & 3-Imix & $10-I \operatorname{mix}$ & $4-I \operatorname{mix}$ & $11 \cdot I_{\operatorname{mix}}$ & $5-I_{\operatorname{mix}}$ & $12-I \operatorname{mix}$ & 6-Imix & 13-Imix & $7-1$ mix \\
\hline $2 \cdot 1 k$ & $9-I k$ & $3 I k$ & $10-J k$ & $4 \cdot / k$ & $11-1 k$ & $5 \cdot 1 k$ & $12-J k$ & $6 \cdot I k$ & $13-1 k$ & $7-I k$ & $1-I k$ & $8-I k$ \\
\hline 3-Akbal & 10. Akbol & 4-Akbal & 11-Akbol & 5-Akbal & 12. Akbal & 6. Akbol & 13-Akbal & 7 -Akbol & 1 -Akbol & 8-Akbal & 2-Akbol & 9-Akbal \\
\hline 4-Kan & $11 \cdot k a n$ & $5 \cdot$ Kan & 12-Kan & 6-kon & 13-Kan & $7-K a n$ & $1-$ Kon & $8-K o n$ & 2 - Kan & $9-K a n$ & $3-\mathrm{Kan}$ & $10-$ Kan \\
\hline 5-Chicchion & 12-Chrection & orchicchion & 13. Chrection & 7.Chicchón & 1. Chicchion & 8.Chicchón & 2-Chicchoin & 9-Chicchion & 3chicethón & 10 Chicchion & 4-Chicchón & 11. Chuschion \\
\hline 6-Cimi & 13. Cimi & 7-C imi & 1. Cimi & 8-Cimi & 2-cims & 9-Cimi & 3-Cimi & 10-Cimi & 4-Cimi & $11-$ cini & 5-Cimi & 12-Cimi \\
\hline 7-Manik & 1-Monik & 8-Monik & 2-Manik & 9-Monith & 3-Manik & 10-Manik & 4-Manik & 11-Monik & 5-Monik & 12-Monik & 6-Manik & 13-Monik \\
\hline 8-Lamat & 2-Lamot & 9-Lomot & 3-Lamat & 10-Lamat & 4-Lamat & 11 -Lamat & 5-Lamat & 12-Lamot & 0 -Lamat & 13-Lamat & 7 -Lamat & 1-Lamat \\
\hline 9-Mulue & 3-Mulue & 10-Muluc & 4-Muluc & 11-Muluc & 5-Muluc & 12-Muluc & 6-Muluc & 13-Muluc & 7-Muluc & 1-Mulue & 8-Muluc & 2-Muluc \\
\hline $10-x$ & $4-O C$ & $11-O c$ & $5 \cdot O C$ & $12-O c$ & $6-0 c$ & $13-0 c$ & $7-O C$ & $1-O C$ & $8-O c$ & $2-0 c$ & $9-O C$ & $3-O C$ \\
\hline 11-Chuen & 5-Chuen & 12-Chwen & 6-Chuen & 13-Chuen & 7-Chuen & 1-Chuen & 8-Chuen & 2-Chuen & 9-Chuen & 3-Chuen & 10-Chuen & 4-Chuen \\
\hline $12 \cdot E b$ & $6 \cdot E b$ & $13-E b$ & $7-E b$ & $1-E b$ & $8-E b$ & $2-E b$ & $9-E b$ & $3-E b$ & $10-E b$ & $4-E b$ & $11-E b$ & $5-E b$ \\
\hline 13-Ben & 7-Ben & 1-Ben & 8-Ben & 2-Ben & $9-B e n$ & 3-Ben & $10-B e n$ & 4-Ben & 11-Ben & 5-Ben & 12-Ben & 6-Ben \\
\hline $1-1 x$ & $8-/ x$ & $2-I x$ & $9-1 x$ & $3-I x$ & $10-I x$ & $4-1 x$ & $11-I x$ & $5-/ x$ & $12-I x$ & $6-1 x$ & $13-I x$ & $7-1 x$ \\
\hline 2-Men & 9 -Men & 3-Men & 10-Men & 4-Men & 11-Men & 5-Men & 12-Men & 6-Men & 13-Men & 7-Men & 1-Men & 8 -Men \\
\hline $3-c i b$ & $10 \cdot \mathrm{Cib}$ & $4-c i b$ & $11-c i b$ & $5-$ cib & $12-c i b$ & $6-c i b$ & $13-c i b$ & $7-c i b$ & $1-C i b$ & $8-c i b$ & $2-c i b$ & $9-c, b$ \\
\hline 4-Caboin & 11-Caboin & 5-Cabain & 12-Caboin & 6-Caboin & 13-Caboin & 7-Cobion & 1-Cabain & 8-Cabain & 2-Coboin & 9 -Coboin. & 3-Cabain & 10-Coboin \\
\hline 5-Exnas & 12-Eznab & 6-Eznab & 13-Eznab & 7-Eznab & $1-E z n a b$ & 8-Eznab & 2-Eznob & 9-Eznab & 3-Eznab & $10-E z n o b$ & $4-\dot{E} z \cap a b$ & 11-Eznob \\
\hline 6-couoe & 13. Couac & 7-Covac & 1-Couac & 8-Couac & 2-Couor & 9-Couoc & 3 Covoc & 10-Covor & 4-caudc & 11 -Cavac & 5-Cauac & 12 Conoc \\
\hline 7-Ahow & 1-Ahou & 8 -Ahav & 2-Ahou & 9-Ahou & 3-Ahow & 10-Ahav & 4-ghou & 11-Ahau & 5-Ahou & 12-Ahou & 6-Ahav & 13-Ahau \\
\hline
\end{tabular}


TABLA 2

\begin{tabular}{|c|c|c|c|c|c|c|c|c|c|c|c|c|c|c|c|c|c|}
\hline 2 & 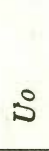 & $i \stackrel{\sim}{\mathbf{N}}$ & $\stackrel{N}{\Delta}$. & U & $\overrightarrow{\mathbb{N}}$ & 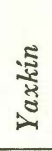 & $\overrightarrow{0}$ & 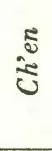 & ఫ్ & N & Uే & z & $\frac{\sqrt[3]{4}}{\sqrt[5]{2}}$ & $\frac{2}{5}$ & జु & $\frac{0}{0}$ & 结 \\
\hline 0 & 0 & 0 & 0 & 0 & 0 & 0 & 0 & 0 & 0 & 0 & 0 & 0 & 0 & 0 & 0 & 0 & 0 \\
\hline 1 & 1 & 1 & 1 & 1 & 1 & 1 & 1 & 1 & 1 & 1 & 1 & 1 & 1 & 1 & 1 & 1 & 1 \\
\hline 2 & 2 & 2 & 2 & 2 & 2 & 2 & 2 & 2 & 2 & 2 & 2 & 2 & 2 & 2 & 2 & 2 & 2 \\
\hline 3 & 3 & 3 & 3 & 3 & 3 & 3 & 3 & 3 & 3 & 3 & 3 & 3 & 3 & 3 & 3 & 3 & 3 \\
\hline 4 & 4 & 4 & 4 & 4 & 4 & 4 & 4 & 4 & 4 & 4 & 4 & 4 & 4 & 4 & 4 & 4 & 4 \\
\hline 5 & 5 & 5 & 5 & 5 & 5 & 5 & 5 & 5 & 5 & 5 & 5 & 5 & 5 & 5 & 5 & 5 & 5 \\
\hline 6 & 6 & 6 & 6 & 6 & 6 & 6 & 6 & 6 & 6 & 6 & 6 & 6 & 6 & 6 & 6 & 6 & 6 \\
\hline 7 & 7 & 7 & 7 & 7 & 7 & 7 & 7 & 7 & 7 & 7 & 7 & 7 & 7 & 7 & 7 & 7 & 7 \\
\hline 8 & 8 & 8 & 8 & 8 & 8 & 8 & 8 & 8 & 8 & 8 & 8 & 8 & 8 & 8 & 8 & 8 & 8 \\
\hline 9 & 9 & 9 & 9 & 9 & 9 & 9 & 9 & 9 & 9 & 9 & 9 & 9 & 9 & 9 & 9 & 9 & 9 \\
\hline 10 & 10 & 10 & 10 & 10 & 10 & 10 & 10 & 10 & 10 & 10 & 10 & 10 & 10 & 10 & 10 & 10 & 10 \\
\hline 11 & 11 & 11 & 11 & 11 & 11 & 11 & 11 & 11 & 11 & 11 & 11 & 11 & 11 & 11 & 11 & 11 & 11 \\
\hline 12 & 12 & 12 & 12 & 12 & 12 & 12 & 12 & 12 & 12 & 12 & 12 & 12 & 12 & 12 & 12 & 12 & 12 \\
\hline 13 & 13 & 13 & 13 & 13 & 13 & 13 & 13 & 13 & 13 & 13 & 13 & 13 & 13 & 13 & 13 & 13 & 13 \\
\hline 14 & 14 & 14 & 14 & 14 & 14 & 14 & 14 & 14 & 14 & 14 & 14 & 14 & 14 & 14 & 14 & 14 & 14 \\
\hline 15 & 15 & 15 & 15 & 15 & 15 & 15 & 15 & 15 & 15 & 15 & 15 & 15 & 15 & 15 & 15 & 15 & 15 \\
\hline 16 & 16 & 16 & 16 & 16 & 16 & 16 & 16 & 16 & 16 & 16 & 16 & 16 & 16 & 16 & 16 & 16 & 16 \\
\hline 17 & 17 & 17 & 17 & 17 & 17 & 17 & 17 & 17 & 17 & 17 & 17 & 17 & 17 & 17 & 17 & 17 & 17 \\
\hline 18 & 18 & 18 & 18 & 18 & 18 & 18 & 18 & 18 & 18 & 18 & 18 & 18 & 18 & 18 & 18 & 18 & 18 \\
\hline 19 & 19 & 19 & 19 & 19 & 19 & 19 & 19 & 19 & 19 & 19 & 19 & 19 & 19 & 19 & 19 & 19 & 19 \\
\hline
\end{tabular}


g) El método para situar una fecha dada, en la Rueda de Calendario, y como secuela de esto, la manera de situar esa misma fecha en la Cuenta Larga, si bien el consumar esta labor requiere la existencia de un dato externo que permita escoger la Serie más adecuada para esa fecha.

\section{Cocientes y COMPLEMENTos}

Entre otras muchas cosas, los cronólogos mayistas de hoy en día conocen, y deben conocer, de memoria, los cocientes de la división, entre 13 y 365 , de los números de días contenidos en los períodos más importantes de la Cuenta Larga.

Por añadidura, conocen y usan a menudo la noción de los complementos al manejar series cíclicas, es decir, que si les parece más fácil y conveniente, cuando se trata de sumar o restar de una cantidad dada una parte de una serie cíclica, prefieren usar el complemento, si es menor, y por consiguiente, de manejo más fácil. Esto es: que si necesitan contar en la Serie Numérica de cualquier período - serie necesariamente compuesta de 13 números - 10 términos hacia adelante, por ejemplo, prefieren contar 3 hacia atrás; pero si los 10 términos han de contarse hacia atrás, el complemento se cuenta hacia ade. lante.

Lo mismo ocurre cuando se trata de avanzar tantos más cuantos lugares desde una posición cualquiera en el Haab: si hay que avanzar 349 lugares, por ejemplo, se prefiere usar el complemento para ajustar 365 días, esto es, 16 con signo negativo, es decir, para contar hacia atrás.

Si los 349 lugares han de restarse, el complemento llevará signo más, quiere decir, se contará hacia adelante.

Pero si nosotros conocemos todo esto y lo usamos a porfía, es más que verosímil que los cronólogos mayas lo conocieran tan bien, o acaso mejor que nosotros y lo usaran en sus cómputos. 
TABLA 3

PERIODOS DE LA CUENTA LARGA

\begin{tabular}{|c|c|c|}
\hline & Periodos & Dias \\
\hline \multirow{15}{*}{. } & Orden $15^{\circ}$ & $1,474,560.000 .000 .000 .000$ \\
\hline & Orden $14^{\circ}$ & $73,728.000 .000 .000 .000$ \\
\hline & Orden $13^{\circ}$ & $3,686,400,000.000 .000$ \\
\hline & Orden $12^{\circ}$ & $184,320,000.000 .000$ \\
\hline & Orden 119 & $9,216,000.000 .000$ \\
\hline & Hablatún & $460,800.000 .000$ \\
\hline & Alautún & $23,040.000 .000$ \\
\hline & Kinchiltún & $1,152.000 .000$ \\
\hline & Calabtún. & 57.600 .000 \\
\hline & Pictún & 2.880 .000 \\
\hline & Baktún & 144,000 \\
\hline & Katún & 7,200 \\
\hline & Tun & 360 \\
\hline & Uinal & 20 \\
\hline & Kin & 1 \\
\hline
\end{tabular}

NOTA: Se incluyen aquí períodos muy superiores, que no dejan de tener interés, si bien la mayor parte de éste es académico, según se echa de ver en el texto correspondiente.

\section{Cálculo de distancias}

El cálculo de distancias entre días del calendario maya, expresados ya por Fórmulas Diurnas, ya por Fechas de Rueda de Calendario, se facilita sobremanera, hasta el punto de que puede hacerse de memoria, con las reglas que deduce uno del análisis de las series de numerales de Kines separados entre sí por $n$ periodos de la Cuenta Larga.

Dichas series, utilizadas en el cálculo de fechas mayas por varios autores (ver Ápenes, 1938; Lizardi R., 1936, 1937, 1939; Merrill, 1942, etc.) se forman dividiendo entre 13 la cantidad de días de los periodos de la Cuenta Larga y utilizando el residuo como razón aritmética.

Puesto que puede uno emplear como punto de origen un 13, es decir, el término final de la serie natural de los guarismos que afectan a los nombres de días, las series dichas quedan como sigue: 


$\begin{array}{lrrrrrrrrrrrrrr}\text { Uinales } & : & 7 & 1 & 8 & 2 & 9 & 3 & 10 & 4 & 11 & 5 & 12 & 6 & 13 \\ \text { Tunes } & \vdots & 9 & 5 & 1 & 10 & 6 & 2 & 11 & 7 & 3 & 12 & 8 & 4 & 13 \\ \text { Katunes } & \vdots & 11 & 9 & 7 & 5 & 3 & 1 & 12 & 10 & 8 & 6 & 4 & 2 & 13 \\ \text { Baktunes } & \vdots & 12 & 11 & 10 & 9 & 8 & 7 & 6 & 5 & 4 & 3 & 2 & 1 & 13 \\ \text { Pictunes } & \vdots & 6 & 12 & 5 & 11 & 4 & 10 & 3 & 9 & 2 & 8 & 1 & 7 & 13 \\ \text { Calabtunes } & \vdots & 3 & 6 & 9 & 12 & 2 & 5 & 8 & 11 & 1 & 4 & 7 & 10 & 13 \\ \text { Kinchiltunes } & \vdots & 8 & 3 & 11 & 6 & 1 & 9 & 4 & 12 & 7 & 2 & 10 & 5 & 13 \\ \text { Alautunes } & \vdots & 4 & 8 & 12 & 3 & 7 & 11 & 2 & 6 & 10 & 1 & 5 & 9 & 13 \\ \text { Hablatunes } & \vdots & 2 & 4 & 6 & 8 & 10 & 12 & 1 & 3 & 5 & 7 & 9 & 11 & 13 \\ \text { Periodo 119 } & \vdots & 1 & 2 & 3 & 4 & 5 & 6 & 7 & 8 & 9 & 10 & 11 & 12 & 13 \\ \text { Periodo 1290 } & \vdots & 7 & 1 & 8 & 2 & 9 & 3 & 10 & 4 & 11 & 5 & 12 & 6 & 13 \\ \text { Periodo 130 } & \vdots & 10 & 7 & 4 & 1 & 11 & 8 & 5 & 2 & 12 & 9 & 6 & 3 & 13 \\ \text { Periodo 14\% } & \vdots & 5 & 10 & 2 & 7 & 12 & 4 & 9 & 1 & 6 & 11 & 3 & 8 & 13 \\ \text { Periodo 150 } & : & 9 & 5 & 1 & 10 & 6 & 2 & 11 & 7 & 3 & 12 & 8 & 4 & 13\end{array}$

El término primero de cada serie, por cierto, es la razón de ella, lo mismo que el residuo de la división, entre 13, del número de días que entran en el periodo de que se trate.

Examinaránse de preferencia las cuatro series primeras, pues las demás tienen muy poco uso.

Desde luego, es conveniente presentar cada una de ellas sin ninguna alteración, poniendo debajo de cada guarismo el intervalo que le corresponde, para luego repetirla en el orden natural de la numeración.

Así se pondrá en relieve un hecho importante:

Que cada numeral está vinculado en cada serie, indefectible e invariablemente, con un intervalo, o distancia, cosa que los cronólogos mayas, a menos de tener una "mente ciega", no pudieron dejar de ver, y de aprovechar en sus cálculos.

Arregladas como queda dicho, las series relativas a los Uinales, es decir, a un Kin que se repite a intervalos de 1.0, son:

$\begin{array}{lrrrrrrrrrrrrrr}\text { Números } & : & 7 & 1 & 8 & 2 & 9 & 3 & 10 & 4 & 11 & 5 & 12 & 6 & 13 \\ \text { Uinales } & : & 1 & 2 & 3 & 4 & 5 & 6 & 7 & 8 & 9 & 10 & 11 & 12 & 13 \\ \text { Sumas } & : & 8 & 3 & 11 & 6 & 1 & 9 & 4 & 12 & 7 & 2 & 10 & 5 & 13 \\ \text { Números } & : & 1 & 2 & 3 & 4 & 5 & 6 & 7 & 8 & 9 & 10 & 11 & 12 & 13 \\ \text { Uinales } & : & 2 & 4 & 6 & 8 & 10 & 12 & 1 & 3 & 5 & 7 & 9 & 11 & 13\end{array}$

Échase de ver desde luego, en el par de abajo:

Que a la parte primera de la serie natural, esto es, a los guarismos 1 a 6 , corresponden pares de Uinales, tantos para cada número como unidades tiene éste, y que a la parte segunda de la misma serie natural, esto es, del 7 al 13, le tocan números impares de Uinales, y por último, que esos números impares equivalen a, o son productos, de multiplicar por 2 cada 
guarismo y de restar luego las trecenas que contengan esos productos.

De aquí sale esta regla dual:

Si se tiene una Fórmula Diurna - FD - y se desea averiguar el intervalo que le corresponde en la serie de los Uinales, multiplíquese el número de esa $\mathrm{FD}$ por 2 y réstese 13 , si es posible. El intervalo encontrado se suma, si se trata de hacer un cómputo hacia adelante desde un punto de partida, pero se resta si el cálculo es hacia atrás.

Si conoce uno el intervalo y desea calcular el número a que corresponde, divide entre 2 ese intervalo, si es par, menor, o mayor de 13; el cociente será el número buscado; pero si es impar se le convierte en par sumándole 13; luego se divide entre 2.

Si se trata de calcular el numeral de FD a que llega uno con ese intervalo, suma al punto de origen, el número encontrado, si el cómputo es hacia adelante; pero lo resta si la cuenta es hacia atrás.

Conviene recordar que conforme a la regla de que al aumento de una unidad en la serie de los Uinales corresponde un intervalo de 2.0, los representados por los guarismos $1,3,5$, $7,9,11$ y 13 son distancias de 14, 16, 18, 20, 22, 24 у 26 Uinales, en el terreno del cálculo nada más, pues en realidad el Tzolkín sólo contiene 13.0.

Una aclaración: en el ámbito de un año, la distancia máxima que se da es de 18.0. Pero la regla que acaba de darse puede aplicarse directamente hasta en caso que el intervalo llegue a 26 Uinales. Más allá será conveniente una de dos cosas: o restar las trecenas posibles, o pasar a la serie de los Tunes o de periodos mayores que el Tun.

Ej. $1^{\circ}$ : Sea el número $10(\mathrm{Ik})$

Multiplicando: $10 \times 2=20$;

Restando 13 tenemos el intervalo buscado: 7.0.

Ej. $2^{\circ}$ : Sea 12 Cabán (O Uayeyab)

Se pregunta cuál es la FD del Cargador.

Hay que regresar 18.0.

Dividiendo entre 2: 9.

Restando 9 de 12, obtenemos 3;

El Cargador es 3 Cabán 
Conviene observar que si se nos dan intervalos superiores a 13.0, para que se determinen los numerales correspondientes, basta con dividir cada uno entre dos, sin restar previamente 13.0, para encontrar el guarismo que le toca:

\begin{tabular}{ccc}
\hline Intervalos & & Numerales \\
\hline 14.0 & Entre 2 & 7 \\
16.0 & $"$ & 8 \\
18.0 & $"$ & 9 \\
20.0 & $"$ & 10 \\
22.0 & $"$ & 11 \\
24.0 & $"$ & 12 \\
26.0 & $"$ & 13 \\
\hline
\end{tabular}

Varios autores (Thompson, 1941: 52; Satterthwaite, 1947: 57, 59, 60, y Guenther Zimmermann, 1935:713) han hecho hincapié muy especial en la utilidad que tiene el usar la regla de los pares de Uinales, es decir, la que indica que a cada dos Uinales varía el numeral de la FD en una unidad, la cual se suma al coeficiente original si se va hacia adelante, o se resta si se retrocede.

En la explicación dada aquí acerca de los intervalos entre las fechas de $\mathrm{RC}$, y sus componentes, resalta igualmente la importancia de aquel hecho.

Todo lo cual podría hacer creer que la regla y el fenómeno que representa son básicos, creencia que no surge directamente del examen de los hechos, los cuales, por cierto, dan a entender que lo básico, lo original, es la variación que van teniendo los numerales, o coeficientes, como se les llama frecuentemente, al repetirse un mismo Kin con intervalos de 1.0. Ni qué decir que la variación es de 7 unidades, que se suman al coeficiente original, si la cuenta es hacia adelante, y que.se restan si el cómputo va hacia atrás. Esas 7 unidades representan la diferencia entre el total de números de la serie de coeficientes y el número de nombres de día.

Volviendo a nuestras series pareadas, hay que decir que guardan una semejanza, si bien leve, con la Tabla 24, A, de Satterthwaite (1947:63), en la cual este autor hace el análisis de los datos relativos al calendario jacalteca recogidos por Oliver La Farge y Douglas Byers (1931). 
La semejanza consiste en el pareamiento de los numerales $1,2,3,4,5,6,7,8$, con los grupos de Uinales $2,4,6,8,10$, 12, 14, 16. Faltan los guarismos 9, 10, 11, 12, 13 .

La Farge y Byers (1931:158) hacen relación de las nociones que en cuanto al año hallaron entre los jacaltecas (Guatemala). Entre esas nociones, la que nos interesa más en este momento es la que explican así los autores ya nombrados:

"La sola subdivisión del año que tiene un nombre es los pies del año, $y$-ok hab:il. Fúndase en un hecho que es obvio si arregla uno una cuenta de días en columnas de cuarenta: que cada vez que aparece por segunda vez un día, tiene un número mayor en una unidad. De esta manera, contando por veintes, tenemos en este año (1927), el Cargador del Año, 7 aH, l. aH, $8 \mathrm{aH}, 2 \mathrm{aH}, 9 \mathrm{aH}, 3 \mathrm{aH}$ y así sucesivamente hasta que habiendo pasado el 13 y habiendo vuelto de nuevo al uno, el año termina en 3 aH. La repetición del Cargador del Año con un número una unidad mayor que al iniciar el año (en este caso 8 aH) es "un pie del año". El siguiente, $9 \mathrm{aH}$, es dos pies, y así sucesivamente hasta que llegamos a $2 \mathrm{aH}$, completando 320 días y ocho pies. Esta cuenta está entonces completa: no se considera que los cuarenta días últimos, que hacen terminar el año en $3 \mathrm{aH}$, agreguen un pie. No se pudo averiguar qué significación tiene esto, si acaso. Fue declarado simplemente, como un hecho matemático interesante".

Puede uno preguntarse si es algo más que un hecho matemático interesante, por ejemplo, si es un método de cálculo. $\mathrm{Y}$ puede preguntarse, asimismo, si es una herencia, entre los jacaltecas, de la sabiduría de los mayas antiguos.

Se inclina uno a aceptar esto, si repara una vez más en lo natural que es en estos cálculos, hacer uso del hecho de que a cada dos Uinales el numeral aumenta en una unidad. Casi puede uno decir que no es fácil hacer este género de cálculos por otro camino que no sea éste.

El suponer que los cronólogos mayas no habían parado mientes en este hecho tan relevante, tan llamativo y tan útil, es suponer, a mi juicio, demasiado.

Por lo tanto, no me parece bien encaminado el escepticismo de Thompson (1941:2) y de Satterthwaite (1947:62). El primero de estos autores, al referirse a la duplicación del número de Uinales cada vez que el numeral de los Kines avanza una unidad, escribe: "Si era desconocida esta fórmula para los ma- 
yas, una tabla del almanaque sagrado, como la fragmentaria del fresco de Uaxactún, daría el mismo resultado, con una molestia muy escasamente mayor". La sola descripción hecha aquí de muchos cálculos cronológicos mayas basta para sospechar vehementemente, que los cronólogos mayas conocieron perfectamente y usaron a porfía, la regla de los Uinales dobles.

Satterthwaite, por su parte, ha dejado escrito en el lugar citado: "He estado desarrollando la idea del Par Uinal por todo lo que vale - tal vez por más de lo que vale".

No creo que haya exagerado.

Las dos series pareadas representan una llave certera para calcular los numerales y los intervalos. Es muy posible que los cronólogos mayas la usaran.

\section{Números de la Serie de Tunes}

Tratándoles como hicimos con los numerales e intervalos de Uinales, tenemos:

$\begin{array}{lrrrrrrrrrrrrrr}\text { Números } & : & 9 & 5 & 1 & 10 & 6 & 2 & 11 & 7 & 3 & 12 & 8 & 4 & 13 \\ \text { Tunes } & : & 1 & 2 & 3 & 4 & 5 & 6 & 7 & 8 & 9 & 10 & 11 & 12 & 13 \\ \text { Sumas } & : & 10 & 7 & 4 & 1 & 11 & 8 & 5 & 2 & 12 & 9 & 6 & 3 & 13 \\ \text { Números } & : & 1 & 2 & 3 & 4 & 5 & 6 & 7 & 8 & 9 & 10 & 11 & 12 & 13 \\ \text { Tunes } & : & 3 & 6 & 9 & 12 & 2 & 5 & 8 & 11 & 1 & 4 & 7 & 10 & 13\end{array}$

La razón en los intervalos túnicos del par segundo de series es 3.0.0. De lo cual inferimos la

Regla: dado un número de FD perteneciente a la Serie de los Tunes, para obtener el intervalo que le toca multiplicamos el guarismo por 3 y restamos las trecenas, si cabe.

Se deduce también, que dado un intervalo de Tunes, para averiguar cuál numeral le toca, se divide directamente por 3 ese intervalo, pero si no es divisible por 3 , se le agregan una o dos trecenas, a manera que la suma sea múltiplo de 3 , y se hace la división.

Ej. 1ํ: Sea el número 7, ligado con cualquier Kin.

Multiplicado por 3 da 21.

Restando 13 quedan 8.

El intervalo es, pues, 8.0.0.

(Recuérdese que todos los números de las Fórmulas Diurnas tienen por punto-cero el 13). 
Ej. $2^{\circ}$ : Tenemos dos series:

$$
\begin{aligned}
& \text { 9.4.19.0.0, } 2 \text { Ahau (3 Xul) y } \\
& \text { 9.4.11.0.0 }
\end{aligned}
$$

Deseamos conocer el número de la FD que corresponde a la segunda.

Vemos sobre la marcha, que el intervalo - distancia- es 8.0.0.

Agregando 13 tenemos 21.

Dividiendo entre 3 llegamos a 7.

Restando este guarismo del 2 (Ahau),

Tenemos: $(13+2)-7=8$

8 (Ahau $3 \mathrm{Mol}$ ) es el número buscado.

Oportuno es observar que si en la serie de los Uinales el paso de $2.0=40$ días, o 40 entre $13=3$ más Residuo 1 , hace aumentar el guarismo de la FD en 1 unidad, aquí, en la serie de los Tunes, ese aumento se logra a cada 3.0.0. o tres pasos, que representan residuos de $9+9+9=27$, que dividido entre 13 deja residuo de 1 .

\section{Serie de los Katunes}

Al formar series análogas a las anteriores con los Katunes observamos que aquí el aumento de 1 Unidad en el numeral es correlativo de un avance de 6.0.0.0, número que dividido entre 13 deja residuo de $6 \times 11=66$. Restando 5 trecenas queda solamente una unidad.

Las series de numerales e intervalos de Katunes, arregladas como las anteriores quedan así:

$\begin{array}{lrrrrrrrrrrrrrr}\text { Números } & : & 11 & 9 & 7 & 5 & 3 & 1 & 12 & 10 & 8 & 6 & 4 & 2 & 13 \\ \text { Katunes } & : & 1 & 2 & 3 & 4 & 5 & 6 & 7 & 8 & 9 & 10 & 11 & 12 & 13 \\ \text { Sumas } & : & 12 & 11 & 10 & 9 & 8 & 7 & 6 & 5 & 4 & 3 & 2 & 1 & 13 \\ \text { Números } & : & 1 & 2 & 3 & 4 & 5 & 6 & 7 & 8 & 9 & 10 & 11 & 12 & 13 \\ \text { Katunes } & : & 6 & 12 & 5 & 11 & 4 & 10 & 3 & 9 & 2 & 8 & 1 & 7 & 13\end{array}$

Las reglas que se deducen son:

Dado un intervalo de Katunes, para encontrar el numeral correspondiente basta con dividir por 6 ; pero si este intervalo no es divisible exactamente entre 6 , se agregan $1,2,3,4$ ó 5 trecenas, a manera que pueda hacerse la división. 
Si lo que se da es el numeral, para encontrar el intervalo basta con multiplicar aquél por 6 y restar las trecenas.

Obsérvese que en las series puestas en el orden del cálculo, las sumas de numeral e intervalo comienzan con 12 y van decreciendo 1 unidad, hasta llegar a 13, que funciona como Cero.

Ej. $1^{\circ}$ : Sea el intervalo 9 (Katunes)

Para hacer la división agregamos tres trecenas:

$9+39=48, y$

$48 \div 6=8$

Ej. $2^{\circ}$ : Se da el numeral 12.

Multiplicando por 6:

$12 \times 6=72$

$72-65=7$

El intervalo es, pues, de 7.0.0.0.

\section{Serie de los Baktunes}

Arreglemos como sabemos ya, los numerales e intervalos de Baktunes, con lo cual podremos observar:

Que al pasar de un guarismo al inmediato, se produce una diferencia de 12.0.0.0.0 si la cuenta es hacia adelante, y de - 12.0.0.0.0 si el cómputo es hacia atrás.

En 12 Baktunes el residuo de la división entre 13 es igual a $12 \times 12=144$.

Restando 11 trecenas, o 143, queda residuo de 1 .

Y ahora, he aquí las series:

Serie de los Baktunes

$\begin{array}{lrrrrrrrrrrrrrr}\text { Números } & : & 12 & 11 & 10 & 9 & 8 & 7 & 6 & 5 & 4 & 3 & 2 & 1 & 13 \\ \text { Baktunes } & : & 1 & 2 & 3 & 4 & 5 & 6 & 7 & 8 & 9 & 10 & 11 & 12 & 13 \\ \text { Sumas } & : & 13 & 13 & 13 & 13 & 13 & 13 & 13 & 13 & 13 & 13 & 13 & 13 & 13 \\ \text { Números } & : & 1 & 2 & 3 & 4 & 5 & 6 & 7 & 8 & 9 & 10 & 11 & 12 & 13 \\ \text { Baktunes } & : & 12 & 11 & 10 & 9 & 8 & 7 & 6 & 5 & 4 & 3 & 2 & 1 & 13\end{array}$

Las reglas son así:

Dado un intervalo de Baktunes, para calcular el número correspondiente, se multiplica ese intervalo por 12 y al producto se le restan las trecenas posibles. Si no hay trecenas, el producto encontrado es el número. 
Si se da el número, el intervalo respectivo se calcula así: se multiplica aquél por 12 y del producto se deducen las trecenas posibles.

Las dos reglas pueden representarse así, respectivamente:

$\mathrm{N}=(\mathrm{I} \times \mathrm{R})-\mathrm{nl3}$, e

$\mathrm{I}=(\mathrm{N} \times \mathrm{R})-\mathrm{n} 13$.

$\mathrm{N}$ es el número, I el intervalo, $\mathrm{R}$ la razón, o 12, y n13 las trecenas que contiene el producto, si acaso.

Estas dos reglas pueden y deben substituirse por ésta:

Para calcular un número cualquiera en una serie de guarismos de Baktunes, dado el intervalo se busca el complemento a 13, de aquel guarismo; si se da el número y se quiere encontrar el intervalo, se busca el complemento a 13, de aquel guarismo.

Ejs.: Intervalo, 7; complemento, 6, que es el numeral buscado.

Numeral, 9: complemento, 4, que es el intervalo deseado.

Una mirada a las series de Baktunes y los numerales basta para comprender que el par formado por cada numeral y su intervalo, suma 13, invariablemente.

Y ello, porque en la serie de los numerales los términos van decreciendo una unidad a cada paso, misma que va aumentando en los intervalos, de modo que lo que se pierde en aquella serie se gana en ésta.

Las operaciones que han de ejecutarse si se aplican las dos reglas dadas renglones atrás son así:

Se da el intervalo, 10.

Multiplicado por 12, el producto es 120 .

Restando las trecenas: $120-117=3$, que es el numeral buscado.

Se da el numeral: 11 .

Multiplicando: $11 \times 12=132$.

Restando las trecenas:

$132-130=2$.

Encontrando residuos, sin dividir

Para encontrar de memoria el residuo de la división entre 13, de la cantidad de Kines contenida en los periodos de la 
Cuenta Larga no es menester, en absoluto, hacer ninguna división.

He aquí el modus operandi, al alcance de cualquier aprendiz de cronólogo mayista, no digamos de un cronólogo maya:

Restando 13, de 20, encontramos el residuo que corresponde a los Uinales: 7.

Multiplicando 7 por 18 , o mejor, por $18-13=5$, tenemos 35. De aquí restamos 2 trecenas y nos queda el residuo de los Tunes: 9.

Multiplicando 9 por 20, o mejor, por $20-13=7$, llegamos a 63.

Restándole 4 trecenas nos queda el residuo correspondiente al Katún: 11 .

Multiplicando $11 \times 20$, o mejor, por $20-13=7$, obtenemos 77 y restando 5 trecenas nos queda el residuo que toca al Baktún: 12 .

Multiplicando 12 por 7 obtenemos 84 y restando 6 trecenas queda el residuo correspondiente al Pictún: 6 .

Multiplicando 6 por 20, o por 7, llegamos a 42 y restando 3 trecenas obtenemos el residuo que toca al Calabtún: 3 .

Y así, sucesivamente.

En conclusión, pienso que si los cronólogos mayas no sabían estas operaciones, verdaderos juegos de niños, no conocían su arte.

Nota: Por supuesto que todas estas reglas pueden substituirse por una:

Si se trata de averiguar cuál es el numeral de un Kin cualquiera al cabo de " $n$ " intervalos expresados en periodos de la Cuenta Larga, multiplíquese " $n$ " por el residuo correspondiente y del producto substráigase el múltiplo mayor de 13 .

Ej. 1: ¿Cuál es el número terminal del Baktún que está 9 Baktunes adelante de 13.0.0.0.0, 4 Ahau?

Multiplíquese: $9 \times 12=108$. Réstese: $108-104=4$.

Súmese este guarismo al punto de partida: 4. Ahau $+4=8$ Ahau.

Ej. 2: ¿Cuál es el numeral del Baktún colocado 8 Baktunes antes que el Baktún 13, 4 Ahau?

Multiplicando: $8 \times 12=96$; restando:

$96-91=5$.

Restando: $4-5$, o $(13+4)-5=12$. 
Ej. $3^{\circ}$ : Si 8.17.0.0.0 es 1 Ahau, ¿cuál Ahau será 8.5.0.0.0?

La diferencia es de 12 Katunes. Multiplicando: $12 \times 11=132$.

Restando: $132-130=2$.

Restando: $1-2$, o $14-2=12$. (Ahau).

$\mathrm{Y}$ así sucesivamente.

\section{Series Numerales de LOS PERIODOS SUPERIORES}

Por más que su importancia es más bien académica, se ha juzgado útil el echar una mirada a la serie de numerales de los periodos superiores de la Cuenta Larga, desde el Pictún - es decir, el sexto-, hasta el $15^{\circ}$, cuyo nombre ignoramos.

Numerales de los Pictunes.-Hemos visto páginas atrás, que el residuo de la división entre 13 , del número de Kines es 6.

Si tomamos un intervalo de 11 Pictunes, tendremos: $11 \times 6=66$, y $66-65=1$.

Es decir, que al avanzar 11 Pictunes, el numeral crece en 1 unidad. Y claro está que si se retrocede, pierde esa misma unidad.

Podemos inferir que si retrocedemos el complemento a 13, o sea 2, el numeral aumentará la misma unidad.

Numerales de los Calabtunes.-Multiplicando el residuo 6, por 7 -es decir, por $20-13$ - tendremos: $6 \times 7=42$, y $42-39=3$, que es el residuo de la división, entre 13, del número de Kines que contiene un Calabtún.

Tomando 9 de éstos tendremos: $9 \times 3=27 ; 27-26=1$.

Es decir, que al avanzar 9 Calabtunes, el numeral avanza una unidad; pero si tomamos el complemento a 13, veremos que se sostiene el aumento de esa unidad. Ese complemento, ni qué decirlo, es 4 Calabtunes.

Numerales de Kinchiltunes.-Multipliquemos el residuo 3, por 20 , o por $20-13=7 ; 3 \times 7=21 ; 21-13=8$, que es el residuo para los Kinchiltunes.

Si le multiplicamos por 5 , tendremos 40 , pero restando tres trecenas quedará 1. Lo cual quiere decir que cada 5 Kinchiltunes, el numeral aumenta en 1 unidad. 
Numerales de los Alautunes.-Multipliquemos el residuo 8 , por 20 , o por $20-13=7$ :

$8 \times 7=56 ; 56-52=4$, residuo de los Alautunes.

Si tomamos 10 de éstos tendremos: $10 \times 4=40$, y $40-39=1$.

Es decir, que si avanzamos 10 Alautunes el numeral crecerá en 1 unidad.

Podríamos preferir el complemento, 3 , y decir que si se retrocede 3 Alautunes, el numeral aumentará en 1 unidad.

Números de los Hablatunes.-Multipliquemos el residuo que corresponde a los Alautunes, por 20, o mejor, por 7:

$4 \times 7=28 ; 28-26=2$, residuo que toca a los $\mathrm{H}_{\mathrm{a}-}$ blatunes.

Sería menester tomar 7 de éstos $-7 \times 2-13=1-$ para lograr un aumento de 1 unidad en el numeral.

Hay que observar que en la Serie de los Hablatunes, los numerales sucesivos van aumentando a cada paso 2 unidades, precisamente lo contrario de lo que hemos visto que ocurre en la Serie de los Katunes.

Números del periodo $11^{\circ}$-Multiplicando el residuo de los Hablatunes por 7 y restando 13, obtenemos 1, que es el residuo en la Serie de los periodos de grado $11^{\circ}$.

Esos números como es de esperar, siguen el orden natural y desde luego, el intervalo necesario para obtener un aumento de 1 unidad en el número es de un periodo.

Números del periodo $12^{\circ}$-Multiplicando el residuo anterior, 1 , por 7 , obtenemos 7 , residuo del periodo de grado $12^{\circ}$, innominado para nosotros.

La serie de números de éste repite fielmente la de los que corresponden a los Uinales. Ni qué decir que a cada dos periodos de éstos, el guarismo aumenta en una unidad.

Números del periodo $13^{\circ}$.-El residuo de este periodo es: $7 \times 7=49$, y $49-39=10$.

Pero $10 \times 4=40$, y $40-39=1$, lo cual significa que a cada 4 periodos de éstos el numeral crece en 1 unidad.

Numerales del periodo $14^{\circ}-\mathrm{El}$ residuo de este periodo es: $10 \times 7=70$, y $70-65=5$. De manera que avanzando 8 de estos periodos el numeral ganará 1 unidad, como sigue: $8 \times 5=40$, y $40-39=1$.

Numerales del periodo $15^{\circ}-\mathrm{El}$ residuo que corresponde a este periodo es: $5 \times 7=35$, y $35-26=9$, de modo que la 
serie de sus numerales repite la de los Tunes, y a cada tres intervalos del periodo $15^{\circ}$ el numeral aumenta en 1 unidad.

\section{ConcLusión}

Esta digresión, que no resultó inútil, hace ver:

Que el residuo correspondiente a cada periodo superior de la Cuenta Larga es igual a la mitad del residuo del periodo inmediatamente anterior; pero si observamos las Series de numerales de Tunes a Baktunes, veremos que allí ocurre lo mismo y que sólo se sale de esta uniformidad la serie de los Uinales, que no es de 20, como los demás periodos, sino de 18. Los residuos son como sigue:

$\begin{array}{lrlr}\text { Uinales } & 7 & \text { Alautunes } & 4 \\ \text { Tunes } & 9 & \text { Hablatunes } & 2 \\ \text { Katunes } & 11 & \text { Grado } 11^{\circ} & 1 \\ \text { Baktunes } & 12 & \text { Grado } 12^{\circ} & 7 \\ \text { Pictunes } & 6 & \text { Grado } 13^{\circ} & 10 \\ \text { Calabtunes } & 3 & \text { Grado 14. } & 5 \\ \text { Kinchiltunes } & 8 & \text { Grado 15 } & 9 \text { etc. }\end{array}$

Que la serie de los numerales de los Hablatunes es inversa de la que toca a los Katunes;

Que los numerales de los periodos de orden $11^{\circ}$ forman una serie inversa de la de los Baktunes;

Que la serie de números del periodo $12^{\circ}$ repite exactamente la de los correspondientes a los Uinales, y por supuesto, que en ella se cumple la regla del Periodo Doble (ver lo relativo al Uinal Doble en pág. 276 ), y

Que la serie que toca al periodo de orden $15^{\circ}$ repite fielmente la de los Tunes y que, como en ésta, un avance de 3 periodos agrega una unidad al numeral, pero que si se retrocede ese intervalo, el numeral merma en una unidad.

¿Por qué ocurre que el intervalo, o residuo, se reduce a la mitad al pasar de una serie de periodos a la que le sigue inmediatamente?

Antes de buscar la explicación hay que aclarar que los guarismos del 1 al 13 no sólo son los acompañantes de los Kines, sino que también fungen como indicadores, o números, de los intervalos y que en la parte del Calendario Maya en que pre- 
valecen las trecenas, la adición o la resta de una o más de esas trecenas no altera en lo mínimo los numerales ni los intervalos, pues el trece funciona a la vez como cero y viene a provocar la repetición de números e intervalos, nada más.

Conviene recordar, asimismo, que la serie original, básica, es la de los Uinales, que viene antes que las demás y de la cual puede uno sacar todas las otras, ya sea por etapas, como se ha hecho aquí, ya sea directamente y aun saltando series intermedias̉.

Hecho este último del cual podemos dar como ejemplo, el siguiente:

Deseamos obtener, desde la serie de numerales de los Uinales, la que toca a los Katunes.

Si recordamos que 1 Katún abarca 360 Uinales y restamos de esta cantidad todas las trecenas que contiene, nos queda un residuo de 9. Este número nos indica el intervalo con que debemos ir escogiendo los numerales de la serie de los Uinales. que es:

$$
7,1,8,2,9,3,10,4,11,5,12,6,13
$$

Partiendo del 13 y contando 9 intervalos unitarios, o números, llegamos al 11, y continuando al mismo paso tenemos la serie Katúnica:

$$
11,9,7,5,3,1,12,10,8,6,4,2,13
$$

$\mathrm{Y}$ ahora entremos en nuestro asunto y tomemos como ejemplo el paso de la serie de los Tunes -intervalo $=9-$ a la de los Katunes.

Hemos encontrado el intervalo katúnico líneas atrás (pág. 283) multiplicando $9 \times 20=180$; restando las trecenas: $180-169=11$.

Éste es la mitad del 9, en la serie de las trecenas, así:

$9+13=22$, y 22 entre $2=11$.

Ahora bien: al multiplicar $9 \times 20$, en rigor hemos multiplicado $9 \times 13$ y $9 \times 7$. El producto primero no nos interesa, pues no altera para nada ni los números ni los intervalos, ya que toda repetición $13^{\mathrm{a}}$ ya en los números, ya en los intervalos, conduce al punto de partida.

Lo que importa es la intervención del 7, el producto de cuya multiplicación puede contener una, o varias trecenas. 
Conviene saber cómo calculamos a primera vista el número de trecenas que forma ese producto, y el residuo correspondiente, para resolver el problema que traemos entre manos.

El producto del 7 por el punto de partida es: $9 \times 7=63$.

No es menester hacer la operación; basta con utilizar la omnipresente regla del Uinal Doble (ver pág. 276), según la cual, una variación de 1 unidad en el numeral corresponde a un intervalo de 2 Uinales; pero si restamos a cada Uinal el 13 tendremos el residuo básico: 7 . El cual, tomado 2 veces, $7+7$ $=14, \mathrm{y}$ restando $13: 14-13=1$.

Esto quiere decir que la Regla del Uinal Doble puede transformarse en la Regla del 7 Doble.

Apliquemos esta última:

En 9 intervalos de la serie de los Uinales hay 4 pares de sietes, es decir, cuatro unidades, más 7 unidades sobrantes: el total es 11 .

Tomemos ahora este 11 como punto de partida para pasar a la serie de los Baktunes:

$11 \times 7=77$, cantidad que contiene cinco trecenas. Restándolas: $77-65=12$.

Q. E. D.

\section{Posición de una Fórmula Diurna en el Tzolkín}

Todo cronólogo mayista conoce de memoria las veinte fórmulas diurnas primeras del Tzolkín, ya que no son más que los nombres de los veinte días, de Imix a Ahau, pareados con la serie de números, del 1 al 13, pero completada ésta con la repetición de los siete números primeros, del 1 al 7 .

Sobre esta base puede calcular sin tablas y sin apuntamientos, la posición de cualquiera Fórmula Diurna en el Tzolkín, conforme a la siguiente

Regla.-Averiguada la Fórmula Diurna con que aparece por vez primera en el Tzolkín el día dado, se saca la diferencia entre su número, o coeficiente, y el de la Fórmula Diurna propuesta; la diferencia se multiplica por $2.0 \mathrm{y}$ al producto se le suma la posición de la Fórmula Diurna inicial del día dado en el mismo Tzolkín. 
Ejemplo:

Deseamos conocer el número de la posición en el Tzolkín, de la Fórmula Diurna 12 Ix.

Ix, día duodécimo, aparece por vez primera en el ciclo de 260 días en la posición 14:, con el numeral 1 .

La diferencia entre 1 y el 12 de la Fórmula Diurna dada es 11 .

Pero $11 \times 2=22$, у $22-13=9$. Es decir, 9.0.

$9.0+14=9.14=194$, es la posición buscada.

\section{Distancia entre dos FóRMUlas diURNaS}

Se supone que pertenecen al mismo Tzolkín o a Tzolkines contiguos.

Deseamos conocer la distancia entre

8 Muluc (Muluc es el día $9^{\circ}$ ) y

6 Cabán (Cabán es el día $17^{\circ}$ )

La diferencia entre 9 y 17 es 8 , número que sumamos a la Fórmula Diurna de partida, 8 Muluc

$$
\frac{\mp 8}{3 \text { Cabán. }}
$$

Comparamos este 3 con el coeficiente de la FD de llegada y tenemos: 3 a 6,3 ; el intervalo es $3 \times 2.0=6.0$. A esto agregamos los 8 días de diferencia y tenemos la distancia buscada:

$$
6.8=128 \text { días. }
$$

De propósito se escogieron estas dos fórmulas diurnas, la primera de las cuales es posterior a la segunda, para hacer ver que esta circunstancia del orden no tiene importancia y por lo tanto, no altera el procedimiento.

Por supuesto que si se nos pide la inversa, es decir, la distancia entre 6 Cabán y 8 Muluc, esperamos que sea igual al complemento de la anterior, es decir: 13.0

$$
\frac{-6.8}{6.12}
$$

En efecto: de Cabán a Muluc hay un intervalo de 12; sumando este número a 6 Cabán llegamos a 5 Muluc. De 5 a 8 
hay 3 , que multiplicado por 2.0 da 6.0 , producto al cual se agregan los 12 días: Muluc.

Tendremos así, 6.12, como distancia entre 6 Cabán y 8

Problema dual que suele presentarse al cronólogo mayista es el de averiguar,

$1^{\circ}$, el numeral que afecta a un Kin que ocupa determinada posición en un año cuyo Cargador se da, y

$2^{\circ}$, el numeral que corresponde al Cargador, si se conoce el que afecta a un Kin cuya posición se da en un año cuyo Cargador se conoce.

Ejemplo del caso primero:

Estamos en un año $1 \mathrm{Ik}$ y deseamos conocer el numeral que afecta a este Kin en 0 Uayeb.

Si aplicamos las reglas expuestas en otra parte de este trabajo (págs. 275-277) podemos proceder como sigue:

El intervalo de 0 Pop a 0 Uayeb es de 18.0. Si restamos 13.0 nos quedan 5.0.

Pero esta distancia corresponde, en la serie de numerales de Uinales sucesivos, a 9.

Sumando este guarismo al del Cargador:

$1+10$ (10 Ik).

Invirtamos el problema:

La FD 10 Ik ocupa la posición 0 Uayeb.

Se desea saber cuál es el Cargador.

Sabemos que el intervalo es de 18.0, correspondientes al guarismo 9, mismo que restamos de 10, con lo cual llegamos a $1 \mathrm{lk}$.

He aquí otros ejemplos, que sirven para mostrar la facilidad de estas operaciones:

Cargador: 4 Cabán.

Se desea saber cuál es la FD que ocupa la posición 0 de Kayab, Uinal $17^{\circ}$.

El intervalo es de 16.0, el cual dividimos entre 2, con lo que obtenemos 8, que sumado al Cargador nos da la resolución.

12 Cabán.

Otro problema: 
La FD 9 Cabán ocupa la posición 0 Muan (Uinal 15). La distancia a 0 Pop es igual a 14.0, o restando 13.0, 1.0.

Aplicando nuestras reglas relativas a los intervalos, o distancias-tiempo que están vinculados a los numerales que afectan a los Uinales sucesivos (págs. 275-276), encontramos que 1.0 corresponde al número 7 .

Restando este guarismo del 9 que figura en la FD dada, tenemos:

2 (Cabán), que es el Cargador buscado.

Si la FD que se nos da no queda en la fila del Cargador, no hay más que retroceder primero, hasta ésta y operar en seguida como queda descrito.

Ejemplo:

Calcular el Cargador del año en que la FD 12 Cib ocupa la posición 9 Pax (Uinal $16^{\circ}$ ).

Restamos 9 de 12 Cib y llegamos a 3 Manik.

Hay que retroceder desde aquí 15.0 , o $15.0-13.0=2.0$.

Pero 2.0 corresponde al numeral 1.

Restando éste de 3, nos queda 2 (Manik), que es el Cargador buscado.

Inversamente:

El Cargador es 7 Cabán.

Se desea saber cuál es el numeral de la FD que ocupa en ese año la posición 13 Mac (Uinal $13^{\circ}$ ).

De 0 Pop a 0 Mac hay una distancia de 12 Uinales; pero 12 corresponde al guarismo 6 , y sumando éste al Cargador: $7+6=13$ (Cabán 0 Mac).

Pero de 0 Mac a 13 Mac hay 13 días, que sumados a 13 Cabán dan 13 Oc.

\section{Calcular la posición de una Fórmula Diurna EN UN AÑO DADO}

Sea la Fórmula Diurna, 9 Ik (Ik es el día $2^{\circ}$ en la lista de los 20), y sea el año, $6 \mathrm{~Eb}$ (Eb es el día $12^{\circ}$ ).

El cronólogo, que sabe de memoria el orden de esos días, no tiene más que buscar el intervalo del primero al segundo: 
10. Suma este número al Cargador $6 \mathrm{~Eb}$ y obtiene $3 \mathrm{Ik}$, la primera Fórmula Diurna con Ik que se presenta en el año dado.

Luego ve que entre los dos numerales de las fórmulas diurnas Ik mencionadas hay una diferencia de 6 unidades, y aplica la Regla del Uinal Doble (ver págs. 275-277), así:

$6 \times 2.0=12.0$.

Agregando 11, posición de $9 \mathrm{Ik}$, se obtiene:

12.11, que es la posición buscada.

Si sumando 13.0 al número de esa posición la suma no pasa de 1.0.5 = $1 \mathrm{Haab}$, ello quiere decir que la Fórmula Diurna dada entra dos veces en el año, cosa que no acontece aquí.

Para que una FD se repita en un año, es menester que la primera aparición ocurra dentro de los 105 días primeros del Haab de que se trate, es decir, de 0 Pop a 4 Xul.

Se dijo renglones atrás, que la posición de $9 \mathrm{Ik}$-en el año $6 \mathrm{~Eb}$, por supuesto - es 11. Lo sabemos, porque estamos enterados de que del Cargador, que ocupa, por supuesto, la posición Cero, a $3 \mathrm{Ik}$, hay una diferencia de 10 días. Y todas las fórmulas diurnas con Ik que entran en este año $6 \mathrm{~Eb}$, ocupan la misma fila que $3 \mathrm{Ik}$, es decir, la undécima.

\section{CÁlCulos FÁCiles de POSICIONES EN EL AÑo}

Se han descrito y discutido aquí algunos arbitrios y reglas que facilitan sobremanera el cálculo de intervalos, o distanciastiempo, entre Fórmulas Diurnas separadas entre sí por grupos de periodos de la Cuenta Larga.

Pasemos ahora a describir y discutir algunos otros, igualmente fáciles, o casi, que sirven para hacer de memoria el cálculo de intervalos y distancias entre posiciones en el año separadas entre sí por grupos de periodos de la Cuenta Larga.

En esta disertación haremos caso omiso de los intervalos expresados en Uinales, por ser el cálculo de ellos, elemental.

Nos ocuparemos, pues, en las distancias expresadas con periodos superiores al Uinal y tocaremos someramente el Pictún, el Cabaltún y otros mayores aún, ya que son de uso tan reducido en las inscripciones, si bien el uso que tienen es de importancia harto crecida. 
Es de advertir desde un principio, que las distancias que se considerarán de preferencia serán aquéllas que estén dentro del ámbito del periodo de la Cuenta Larga que se considere, puesto que las distancias que desborden ese límite pueden y deben considerarse en los periodos mayores. Quiere decir esto, que una distancia superior a 19.0.0 cae naturalmente, en el ámbito, o marco, de la serie de los Katunes; que una mayor de 19.0.0.0, entra en la esfera de la Serie de los Baktunes y que una superior a 19.0.0.0.0 ha de analizarse en el nivel de los Pictunes, y así sucesivamente. Lo cual no quiere decir que no sea dable el calcular una distancia expresada con un número desusado de Tunes, pongamos por caso, 500. Puédese hacer esto, pero se preferirá reducir el número, a unidades superiores, así:

\subsubsection{0 y operar con esta serie.}

Luego de hacer relación de los procedimientos y reglas pertinentes, propondráse una reflexión acerca de si los mayas antiguos fueron capaces, o no, de llevar al cabo lo que ejecutan hoy día con harta facilidad los cronólogos mayistas, quiere decir, los estudiosos que discuten, manejan y aplican un sistema ajeno a su civilización, y a veces, muy alejado del modo peculiar de pensar que tienen ellos.

\section{La Regla Relativa a Los tUNes}

Nadie que dedique algún tiempo y atención a la Cronología maya dejará de advertir el hecho, por demás notorio, de que la división y la resta que se dan aquí dejan un residuo de 360 la primera y de 5 la segunda:

$$
\frac{360}{365} \cdots 360, \text { y } 360-365=-5 .
$$

Y siendo fácil hasta la puerilidad el sumar 5, o restar 5, o multiplicar por 5 , dicho se está que a cualquiera que campee en este viejo campo de la Cronología maya se le ocurrirá usar esa diferencia, o ese residuo, 360 , o su complemento, -5 , al calcular el cambio de posición anual a intervalos de Tunes.

Por tanto, si se le propone el problema de calcular cómo varía una posición en el año después de sumar, o de restar, quiere decir, de avanzar, o retroceder, desde una posición 
dada, determinada distancia expresada en Tunes, no dejará de utilizar aquel guarismo.

Aclaremos de una vez por todas que nuestro 5 es negativo, es decir, -5 , porque es lo que le falta al Tun para igualar al año, esto es, porque es el complemento, para 365 , de 360 días que tiene el Tun.

Ello significa que si la cuenta es hacia adelante, hay que restar. Y claro se ve que si el cómputo es hacia atrás, hay que invertir la operación: debe uno sumar.

Aclarado esto, venga un

Ejemplo: De la posición anual $2 \mathrm{Xul}$, retroceder 19.0.0.

Para dar mayor realidad al ejemplo, escojamos una Serie con Fecha de Rueda de. Calendario que contenga nuestra posición anual:

(8. 14. 4. 0.19, 10 Cauac) 2 Xul. Restando los 19 Tunes: $-19.0 .0$

8. 13. 5. 0.19 (8 Cauac $17 \mathrm{Yax}$ ).

He aquí las operaciones:

$19 \times 5=95=4.15$.

Si se avanza, ese total debe llevar signo menos, así: - 4.15.

Pero como hay que retroceder, ese total debe llevar signo contrario, y queda así: 4.15, quiere decir, hay que sumarlo.

Sumando, pues, a 2 Xul -Uinal $6^{\circ}-$, 4.0, llegamos al Uinal 10-, Yax, así: 2 Yax, y agregando los 15 Kines, alcanzamos la posición 17 Yax.

Cuanto a la Fórmula Diurna ya sabemos cómo encontrarla (ver págs. 274 y sigs.).

La resolución es:

$$
\text { (8 .13. 5. 0.19, } 8 \text { Cauac) } 17 \text { Yax. }
$$

Debe uno reconocer que si el intervalo contiene también Uinales y Kines, la operación se complica, pero no mucho,y que se realizará con felicidad si procede uno por etapas, calculando primero el cambio de posición causado por la resta, o suma, del Número-Distancia expresado en periodos completos, del tercero arriba, y luego, sumando, o restando, los Uinales y Kines, cuya suma no pasará nunca de 1.0.0. 
AÑo de Cálculo, o AÑo Ritual

En la clase de los arbitrios y procedimientos que usaron los cronólogos mayas en sus cómputos calendáricos entra el que Eric Thompson (1941:43-47) llama "año de cálculo" -364 días- muy útil y cómodo para aquellas operaciones, porque es múltiplo exacto de 13, lo cual significa que dos Fórmulas Diurnas separadas una de otra por un "año" de éstos llevan el mismo número, si bien su día cambia, por causa de los cuatro en que el "año" de 364 días excede al Tun.

Todo lo cual quiere decir que el Kin de la Fórmula Diurna de llegada será el $4^{\circ}$ después del Kin de la Fórmula Diurna de partida, cuando la cuenta sea hacia adelante.

Si, por ejemplo, se pregunta a cuál Fórmula Diurna llegamos agregando 364 días a 12 Ahau, responderemos:

$12 \mathrm{Kan}$.

Empero, si la cuenta es para atrás, la respuesta será: 12 Ahau $-364=12$ Cib.

Thompson (1941:48) ha hecho ver que al operarse con la unidad superior inmediata al "año de cálculo", $364 \times 20$ $=7,280$ días, las operaciones resultan igualmente fáciles, ya que no cambian el día y su número, y que el deslizamiento en el Haab es de un Uinal.

Este deslizamiento es para atrás si se suma la unidad de orden segundo a que venimos refiriéndonos, $364 \times 20$, pero es para adelante si se substrae esa misma unidad.

El autor inglés da estos ejemplos:

9.10.10. 0. 0, 13 Ahau 18 Kankín

+ 1. 0.4. $0(364 \times 20)$

9.11.10. 4. 0, 13 Ahau $18 \mathrm{Mac}$ (Mac es el Uinal que precede inmediatamente a Kankín).

9.14.19. 5. 0, 4 Ahau 18 Muan

-3.0.12. $0 \quad(364 \times 20 \times 3)$

9.11.18.11. 0, 4 Ahau 18 Cumkú. (Cumkú está tres Uinales adelante de Muan)

La repetición de la Fórmula Diurna al cabo de $364 \times 20$ $=7,280$ días, débese a que esta cantidad es múltiplo exacto de 260 , suma que está contenida en ella 28 veces. 
Hay que recordar que el número 91, citado también en el Códice de Dresden, es la cuarta parte de 364, y por supuesto, múltiplo exacto de 13: $7 \times 13=91$.

Por lo tanto, las Fórmulas Diurnas separadas entre sí por distancias de 91 días o sus múltiplos, contienen el mismo número, o coeficiente.

Las listas de múltiplos de 91 y 364 están en las láminas 32a, 45a, 63 y 64 del Códice de Dresden y sus partes faltantes han sido reconstruidas por Thompson (1941: Figs. 1, 2).

Förstemann (1906:133) llama "año ritual" el grupo de 364 días. Spinden (1924:62) le da el mismo nombre y dice que pudo haber sido escogido como medida del tiempo sideral, primariamente, por razones matemáticas.

Thompson (1941:52-57) muestra cómo pudo usarse el "año de cálculo" para fijar fechas de Rueda de Calendario en la Cuenta Larga y cómo los Números-Anillo de la Lámina 31a del Dresden y ciertos yerros en las inscripciones, tienden a confirmar que aquel periodo - 364 días - fue usado por los "astrólogos" mayas para calcular fechas.

\section{REgLa DE LOS KATUNES TRIPLE, CUÁDRUPLE, SÉPTUPLE, ÓCTUPLE Y DÉCUPLE}

Para formularla, presentemos los diez múltiplos primeros, con sus complementos, cuando sea necesario, de 265 , residuo de la división, entre 365 , de los 7,200 días que componen el Katún:

TABLA 4

\begin{tabular}{ccc}
\hline Múltiplos & Días y complementos & Notación maya \\
\hline 19 & 265 & 13.5 \\
& -100 & -50 \\
$2^{\circ}$ & 165 & 8.5 \\
& -200 & -10.0 \\
39 & 65 & 3.5 \\
$4^{9}$ & -35 & -1.15 \\
$5^{9}$ & 230 & 11.10 \\
& -135 & -6.15 \\
$6^{9}$ & 130 & 6.10 \\
79 & 30 & 1.10 \\
$8^{\circ}$ & -70 & -3.10 \\
99 & 195 & 9.15 \\
$10^{\circ}$ & 95 & 4.15 \\
\hline
\end{tabular}


Sin esfuerzo echa uno de ver que los múltiplos más pequeños, y por ello mismo más manejables, son: el tercero, el cuarto, el séptimo y el décimo.

Cada quien puede escoger el que guste; por lo que hace al autor de este Resumen, ha usado con mayor ventaja, el múltiplo tercero.

Usémosle en los ejemplos que siguen:

Ej. 1: Desde 8.0.0.0.0, 9 Ahau 3 Zip, avanzar 18.0.0.0. En 18 Katunes hay 6 grupos de 3 . El residuo final es: $65 \times 6=390$, menos 365 , quedan $25=1.5$. Si a 3 Zip (Uinal $3^{\circ}$ ) le sumamos 1. 0. llegamos al Uinal $4^{\circ}: 3$ Zotz.

Sumando los 5 días faltantes obtenemos 8 Zotz.

Resolución: 8 .18. 0. 0. 0, 12 Ahau 8 Zotz.

Ej. 2\%: 9.17. 0. 0. 0, 13 Ahau 18 Cumkú

-15 . 0. 0.0

9. 2. 0.0 .0

En 15 Katunes hay 5 grupos de 3 Katunes:

$65 \times 5=325=16$. 5. Usando el complemento: $-40=-2.0$.

Como hay que retroceder, la cantidad cambia de signo, es decir, se suma:

18 Cumkú (Uinal 18 $\left.{ }^{\circ}\right),+2.0=13$ Uo (Uinal $2^{\circ}$ )

Resolución: 9. 2. 0. 0. 0, (4 Ahau) 13 Uo.

Los paréntesis puestos a la Fórmula Diurna indican que ésta se calcula como ya está explicado en otra parte.

Ej. $3^{\circ}$ : 8.17. 0. 0. 0, 1 Ahau 8 Chen

$-16.0 .0 .0$

8. 1. 0. 0.0

En 16 Katunes hay cinco grupos de tres, más una unidad.

El residuo es, pues:

$(5 \times 65)+265=590$,

Restando 1 año $\begin{aligned} & -365 \\ & 225=11.5 .\end{aligned}$ 
Retrocediendo 11 Uinales desde 8 Chen (Uinal $9^{\circ}$ ), llegamos al Uinal $16^{\circ}$ : $13 \mathrm{Pax}$, y retrocediendo 5 días, tenemos $8 \mathrm{Pax}$.

La resolución es: 8. 1. 0. 0. 0 (7 Ahau), 8 Pax. Se aconseja usar de preferencia los complementos, si son menores que los residuos.

Aquí, el complemento es 7. 0, con signo menos, pero como se avanza, hay que sumarlo.

$\mathrm{Al}$ operar con intervalos de Katunes conviene tener presente que puede uno usar el complemento de 265, o sea - 100, cantidad ésta que se resta si avanza uno, y se suma, si se retrocede.

En el ejemplo que acabamos de ver tendríamos:

$16 \times-100=-1600$, y restando cuatro años:

$-1600$

$-1460$

$\overline{-140}=-7.0$, que cambia de signo, pues la cuenta es hacia atrás: 8 Chen $+7.0=8$ Pax.

\section{LA REGLA DEL BAKTÚN DOBLE}

Tenemos un arbitrio muy fácil y cómodo para calcular de memoria los cambios de la posición en el año en la serie de los Baktunes. Se funda en que cada 2 Baktunes la posición en el Haab se desliza 15 días hacia adelante si la cuenta es en ese sentido, hacia atrás si el cómputo es de resta.

La explicación está en que siendo 190 el residuo de la división entre 365 , de los 144,000 días que forman un Baktún, el residuo de 2 Baktunes es: $190 \times 2=380$, y $380-365$ $=15$.

Este arbitrio es análogo al del Uinal Doble, tan útil en el cálculo de distancias entre Fórmulas Diurnas.

El residuo de 4 Baktunes es 30 días; el de 8 Batunes, 60 días, o 3.0, y el de 9 Baktunes, $60+190=250$, o el complemento a 365 , de éste: $-115=-5.15$.

Ej. 1ํ: Desde 13.0.0.0.0, 4 Ahau 8 Cumkú, avanzar 9.0.0.0.0 
TABLA 5

INTERVALOS EN PERIODOS DE LA CUENTA LARGA

RESIDUOS DE LA DIVISIÓN $\div 365$

MÚLTIPLOS PARA CALCULAR POSICIONES EN EL AÑO

\begin{tabular}{|c|c|c|c|c|c|c|c|c|c|c|}
\hline Periodos & $\begin{array}{l}\text { Residuos } \\
\quad \div 365\end{array}$ & Múltiplo $2^{\circ}$ & Múltiplo $3^{\circ}$ & Múltiplo 49 & Múltiplo 5 & & Múltiplo $7^{\circ}$ & Múltiplo 89 & Múltipl & $10^{\circ}$ \\
\hline 3?-Tun & $\begin{array}{r}360=18.0 \\
-\quad 5=-.5\end{array}$ & & & & & & & & & \\
\hline $4^{0}$-Katún & $\begin{array}{r}265=135 \\
-100=-5.0\end{array}$ & $165=8.5$ & $65=$ & $\begin{aligned} 330= & 16.10 \\
-35= & -1.15\end{aligned}$ & & & $30=1.10$ & $-70=-3.10$ & $95=$ & 4.15 \\
\hline 5?-Baktún & $190=$ & $15-.15$ & & & & & & & & \\
\hline 6:-Pictún & $150=7.10$ & $\begin{array}{r}300=15.0 \\
-65=-3.5\end{array}$ & & & & & & & & \\
\hline 7a-Calabtún & $80=\quad 4.0$ & . & $\begin{array}{r}240=12.0 \\
-125=-6.5\end{array}$ & $\begin{aligned} 320 & =16.0 \\
-45 & =-2.5\end{aligned}$ & $35=$ & 1.15 & & & & \\
\hline 89-_Kinchiltún & $140=7.0$ & $\begin{array}{r}280=14.0 \\
--85=-45\end{array}$ & $55=$ & & & & & & & \\
\hline 9?-Alautún - & $\begin{array}{r}215=12.5 \\
-120=-6.0\end{array}$ & $125=6.5$ & 5 & $\begin{aligned} 250 & =1210 \\
-115 & =-5.15\end{aligned}$ & & & & & & \\
\hline 100-Hablatún & $155=\quad 7.15$ & $\begin{aligned} 310 & =15.10 \\
-55 & =-2.15\end{aligned}$ & $100=$ & & & & & & & \\
\hline $11 \%$ & $180=9.0$ & $\begin{aligned} 360 & =18.0 \\
-\quad 5 & =-\quad .5\end{aligned}$ & & & & & . & & & \\
\hline $12 ?-$ & $\begin{aligned} 315 & =1515 \\
-50 & =-2.10\end{aligned}$ & $-100=-5.0$ & $-150=-7.10$ & & & & & & & \\
\hline $13 \%$ - & $95=4.15$ & & $\begin{aligned} 285 & =14.5 \\
-80 & =-4.0\end{aligned}$ & & & & & & & \\
\hline $\begin{array}{l}14 \%- \\
15 \%-\end{array}$ & $\begin{array}{l}75= \\
40=\end{array}$ & & & & $10=$ & .10 & & & $20=$ & 1.0 \\
\hline
\end{tabular}


Según la cuenta hecha renglones atrás, hay que retroceder, primero, 5.0, desde 8 Cumkú -Uinal $18^{\circ}$-, con lo cual llega uno a la posición $9^{\text {a }}$ del Uinal $13^{\circ} ; 8$ Mac. Luego retrocedemos 15 días, y llegamos a 13 Ceh.

Con las reglas que ya conocemos calculamos la FD y tenemos:

$$
\text { 9.0.0.0.0, } 8 \text { Ahau } 13 \text { Ceh. }
$$

Ej. $2^{\circ}$ : Desde 11.0.0.0.0, 6 Ahau 8 Mac, retroceder 10.0.0.0.0

Hay aquí cinco pares de Baktunes, correspondientes a un residuo de 75 días $=3.15$, que debemos restar, ya que la cuenta es hacia atrás.

Restando a 8 Mac -Uinal $13^{\circ}$ - 3.0, llegamos al Uinal $10^{\circ}$, y tenemos: 8 Yax. Restando los 15 días llegamos a 13 Chen.

La resolución es: 1.0.0.0.0 (3 Ahau), 13 Chen.

Ej. $3^{\circ}$ : Retroceder 13.0.0.0.0, desde

$$
\text { 13.0.0.0.0, 4. Ahau } 8 \text { Cumkú. }
$$

El residuo es 6 (Pares de Baktunes $) \times 15=90$; y $90+$ $190=280$.

Tomando el complemento a 365 , igual a $-85=-4.5$, y retrocediendo - 4.0, es decir, avanzando 4.0, pues aquel número cambia de signo al hacerse la cuenta hacia atrás, tenemos:

8 Cumkú (Uinal 18 $8^{\circ}$, más $4.0=3$ Zotz (Uinal $4^{\circ}$ ). Sumando los 5 Kines: 8 Zotz.

La resolución es: 13.0.0.0.0, 4 Ahau 8 Zotz.

Esta fecha, por cierto, es el punto cero de la Serie Inicial del Tablero del Templo de la Cruz, Palenque, Chiapas.

En conclusión, tenemos la Regla siguiente:

Si deseamos calcular el cambio de posición en el Haab en la serie de los Baktunes, contamos los pares de Baktunes que haya y su total se multiplica por 15; si sobra un Baktún, representa una diferencia de 190 días, la cual se suma al producto anterior.

La suma de los residuos se suma al punto de partida si la cuenta va hacia adelante, pero se resta si la cuenta va hacia atrás. 


\section{Fijar la posición de una Fecha en La RUEDA DEL CALENDARIO}

Es ésta una operación que puede hacerse de memoria, en tres etapas:

$1^{\text {a }}$ Fijar la posición de la fëcha, en el año.

$2^{\text {a }}$ Encontrar el Cargador del año en que cae la fecha.

$3^{a}$ Determinar la posición de ese año en el ciclo de 52 años.

Operaciones primera y segunda:

Sea la Fecha de Rueda de Calendario 4 Ahau 8 Cumkú, formada por el día $20^{\circ}$, la posición $9^{a}$ en el Uinal y el Uinal $18^{\circ}$, datos que nos sirven para reconocer ipso facto, que han pasado 17 Uinales del año y 9 días, contando éstos como tiempo vencido.

La posición en el año es, pues: 17.9, o sea la $349^{\text {a }}$.

Para calcular el Cargador retrocedemos 348 días, hasta 0 Pop, desde cada uno de los tres componentes de la fecha: 4./Ahau/8 Cumkú.

Empecemos con el 4: buscamos mentalmente el múltiplo máximo de 13 deducible de las dos cifras primeras -34- y vemos que es 26 y que el residuo es 8 ; agregando lás 8 unidades tenemos 88 y descontando el múltiplo mayor de 13 , que es 78 aquí, nos quedan 10. Restando éste de 4, o mejor dicho, de $13+4$, nos quedan 7 .

Pasemos al día dividiendo: 348 entre 20 obtenemos un residuo de 8 , y retrocediendo 8 pasos desde Ahau, día $20^{\circ}$, llegamos al día $12^{\circ}$ : Ben.

El Cargador es 7 Ben.

Operación tercera:

Aceptando convencionalmente como principio de la Rueda de Calendario el año $1 \mathrm{Ik}$, esa Rueda queda así: 
TABLA 6

\begin{tabular}{|c|c|c|c|c|c|c|c|c|}
\hline \multicolumn{2}{|c|}{ Tlalpilli Ik } & \multicolumn{2}{|c|}{ Tlalpilli Manik } & \multicolumn{2}{|c|}{ Tlalpilli $E b$} & \multicolumn{3}{|c|}{ Tlalpilli Cabán } \\
\hline 1. & $1 \mathrm{Ik}$ & 1. & 1 Manik & 1. & $1 \mathrm{~Eb}$ & 1. & 1 Cabán & \\
\hline 2. & 2 Manik & 2. & $2 \mathrm{~Eb}$ & 2. & 2 Cabán & 2. & $2 \mathrm{Ik}$ & \\
\hline 3. & $3 \mathrm{~Eb}$ & 3. & 3 Cabán & 3. & $3 \mathrm{Ik}$ & 3. & 3 Manik & \\
\hline 4. & 4 Cabán & 4. & $1 \mathrm{Ik}$ & 4. & 4 Manik & 4. & $4 \mathrm{~Eb}$ & \\
\hline 5. & $5 \mathrm{Ik}$ & 5. & 5 Manik & 5. & $5 \mathrm{~Eb}$ & 5. & 5 Cabán & \\
\hline 6. & 6 Manik & 6. & $6 \mathrm{~Eb}$ & 6. & 6 Cabán & 6. & $6 \mathrm{Ik}$ & \\
\hline 7. & $7 \mathrm{~Eb}$ & 7. & 7 Cabán & 7. & $7 \mathrm{Ik}$ & 7. & 7 Manik & \\
\hline 8. & 8 Cabán & 8. & $8 \mathrm{Ik}$ & 8. & 8 Manik & 8. & $8 \mathrm{~Eb}$ & \\
\hline 9. & $9 \mathrm{Ik}$ & 9. & 9 Manik & 9. & $9 \mathrm{~Eb}$ & 9. & 9 Cabán & - \\
\hline 10. & 10 Manik & 10. & $10 \mathrm{~Eb}$ & 10. & 10 Cabán & 10. & $10 \mathrm{Ik}$ & \\
\hline 11. & $11 \mathrm{~Eb}$ & 11. & 11 Cabán & 11. & $11 \mathrm{Ik}$ & 11. & 11 Manik & \\
\hline 12. & 12 Cabán & 12. & $12 \mathrm{Ik}$ & 12. & 12 Manik & 12. & $12 \mathrm{~Eb}$ & \\
\hline 13. & $13 \mathrm{Ik}$ & 13. & 13 Manik & 13. & $13 \mathrm{~Eb}$ & 13. & 13 Cabán & \\
\hline
\end{tabular}

Una inspección atenta de la Tabla 6 basta para deducir las cuatro reglas siguientes, cuyo conocimiento nos habilitará para determinar de memoria, la posición, en la Rueda de Calendario, de cualquiera de los 52 años que la componen.

Regla primera.--Si el número del año es cualquiera de los impares 1, 5, 9, 13, el Haab pertenece al Tlalpilli, o Trecena, de su nombre.

Regla segunda.-Si el número del Haab es cualquiera de los impares $3,7,11$, el año pertenece a un Tlalpilli posterior en dos Tlalpillis al de su nombre.

Regla tercera.-Si el número del año es alguno de los pares 2, 6, 10, el Haab queda en un Tlalpilli posterior en tres Tlalpillis al de su nombre. Usando el complemento a 4, podemos decir: en un Tlalpilli anterior en una Trecena al de su nombre.

Regla cuarta.-Si el número del Haab es alguno de los pares $4,8,12$, el año corresponde a un Tlalpilli posterior en una Trecena al de su nombre.

Volviendo a nuestro problema después de esta digresión esencial, diremos que debemos aplicar aquí la Regla segunda, la cual nos lleva a reconocer que el año $7 \mathrm{~Eb}$ es el séptimo de la Rueda y que hasta la fecha 4 Ahau 8 Cumkú van transcurridos, contando tiempo vencido: 


$$
6 \text { años }+ \text { (349 días, o 17.9). }
$$

Como hacemos de memoria las operaciones descritas aquí, debemos usar en este momento el método más sencillo para convertir los años, en Tunes, método que consiste en agregar al número de los años, cinco días por cada uno de ellos, o sean 30 días $=1.10$.

La notación vigesimal es:

$6.1 .10+17.9=7.0 .19$, que da la posición de 4 Ahau 8 Cumkú en la Rueda de Calendario que convencionalmente hacemos principiar en el año $1 \mathrm{Ik}$.

\section{BIBLIOGRAFfA}

Árenes, Ola. 1937. Table for Determination of Maya Calendar Round Positions. Ethnos, vol. 2, núm. 4, pp. 97-101. Estocolmo, Suecia.

— 1939. Tabla de lectura directa para el cálculo de fechas mayas. Revista Mexicana de Estudios Antrpológicos, pp. 54-61, tomo III, núm. 1, enero-abril. (Conferencia leída ante la Sociedad Mexicana de Antropología en la sesión ordinaria del día 20 de enero de 1938). México, D. F.

Förstemann, Ernst. 1906. Commentary on the Maya Manuscript in the Royal Public Library of Dresden. Papers of the Peabody Museum of American Archaeology and Ethnology, Harvard University, vol. Iv, núm. 2, pp. 267. Cambridge, Mass. Octubre.

La Farge, Oliver II y Douglas, Byers. 1931. The Year Bearer's People. The Tulane University of Louisiana. Middle American Research Series Publication, núm. 3. Nueva Orleans.

Lizardi Ramos, César. 1936. Recurrencias de las fechas mayas. México, D. F.

1937. El orden de los katunes de la Cuenta Corta.

1939. Cómputos de fechas mayas. Actas de la primera sesión celebrada en la ciudad de México en 1939, del Vigesimoséptimo Congreso Internacional de Americanistas. Instituto de Antropología e Historia, vol. I, pp. 356-359. México, D. F.

Merrill, Robert Hall. 1942. Mechanically Counting by Fives. Maya Style. Copias mimeográficas del resumen del trabajo sometido el 8 de mayo de 1942 a la Sociedad para la Arqueología Americana. Grand Rapids, Mich. 
Satterthwaite, Linton, Jr. 1947. Concepts and Structures of Maya Calendrical Arithmetics. Publicacion del Museum of the University of Pennsylvania and The Philadelphia Anthropological Society, núm. 3. Filadelfia, Penn.

Spinden, Herbert J. 1924. The Reduction of Mayan Dates. Papers of the Peabody Museum of American Archaeology and Ethnology, Harvard University, vol. vi, núm. 4. Cambridge, Mass.

Teeple, John E. 1937. Astronomía maya. Anales del Museo Nacional de Arqueología, Historia y Etnografía, tomo II, pp. 479-581. (EI pie de imprenta de ese volumen lleva el año 1935, pero en el colofón se da el 1937). La separata, o el sobretiro, a que se refieren las citas hechas en este artículo, lleva numeración especial: 7-109.

Thompson, J. Eric S. 1941. Maya Arithmetic. Contributions to American Anthropology and History, núm. 36. Carnegie Institution of Washington, Publicación 528, pp. 37-62, marzo $1^{\circ}$. Washington, D. C.

ZimmermanN, Günther. 1935. Einige Erleichterungen beim Berechnen von Maya-Daten. En Anthropos, tomo xxx, pp. 707-715. St. Gabriel Mödling bei Wien, Österreich. 